\title{
The hunt for neutrino mass (cont'd)
}

\author{
New measurements deny an earlier report of a neutrino mass of $17.1 \mathrm{keV}$. But the hunt will go on. One \\ benefit will be more accurate measurements of decay energy-spectra.
}

THE hunt for the heavy neutrino continues, with mounting ingenuity but without success. So much is clear from the latest experiment, reported by a group from the physics department at Princeton University (Altzitzoglou, T. et al., Phys. Rev. Lett. 55, 799; 1985). The group's conclusion is that there is no evidence from the electron decay of sulphur-35 $\left({ }^{35} \mathrm{~S}\right)$ for the presence of neutrinos with non-zero mass among the decay products. But given the compatibility of heavy neutrinos with present particle theories including the Salam-Weinberg electroweak theory, the tantalizing reports that heavy neutrinos may indeed be real and their attractiveness in other fields, cosmology in particular, it is unlikely that Altzitzoglou et al. have spoken the last word on the subject.

For the time being, $\beta$-decay seems to have become the preferred phenomenon, if only because it allows human-scale experiments to be designed. The basis of these investigations remains substantially what it was in the 1930 s, when Fermi postulated the existence of a neutrino with zero mass, but otherwise with the properties of electronic matter (leptons), to accommodate the observed energy spectra of the electrons (ranging from nothing to a maximum) so as to allow energy and linear and angular momentum (spin) to be conserved.

The difference now is that there are at least three generations of neutrinos, one corresponding to each of the three known leptons (muons and tauons as well as electrons), and that even if the electron neutrino (strictly, it is an anti-neutrino) should have zero mass, the theories require that all three neutrinos (but there may be more) should be mixed together in the sense of quantum mechanics in the products observed.

The first experiment designed to look for non-zero mass was reported by a Soviet group nearly a decade ago, and turned on the measurement of the energy spectrum of the electrons from the $\beta$ decay of tritium. The result reported, a non-zero mass, has served chiefly as a reminder of the formidable difficulty of making convincing measurements in this field. The essential problem is that neutrinos with non-zero mass will affect the energy-spectrum of the electrons emitted in $\beta$-decay only in the region of electronenergy corresponding to the supposed mass, which cannot be more than a few electron-volts without being more of an embarrassment than a comfort to the cosmologists. In short, the hunt for neutrinos with non-zero mass must be carried out just in that region where accurate measurements are most difficult.

Experimentalists are clearly undismayed by this challenge. Thus, earlier this year, J.J. Simpson, from the University of Guelph in Canada, described a repetition of the Soviet measurement by the intriguing technique of embedding radioactive tritium atoms in a slice of silicon, in which decay electrons can be counted as pulses of electric current, and where their total energy can be measured directly (Phys. Rev. Lett. 54, 1891; 1985).

Simpson's conclusion was that the shape of his electron energy-spectrum was indeed distorted from that expected on the assumption that all neutrinos have zero mass; at the low end of the spectrum, there was an excess of electrons, most simply explained by a redistribution of the total energy shared by the products of the $\beta$-decay between the electron and the neutrino. His result was that at least one of the three neutrinos has non-zero mass, that the most probable value of that mass is $17.1 \mathrm{keV}$ and that the quantum state representing the heavy neutrino constitutes some 3 per cent of that observed in experiments, manifested by the decay electrons.

As in true love, the path of discovery is never quite smooth. No doubt inspired by the uncomfortably large neutrino mass reported by Simpson, W.C. Haxton from the University of Washington and Los Alamos has already put forward one reason for believing that the result may be an artefact of interpretation (Phys. Rev. Lett. 55, 807; 1985). Simpson's result hangs on the difference between the measured spectrum and that predicted in calculations with neutrinos of zero mass.

Haxton argues that Simpson's expectations may be oversimple approximations. His argument may be incomplete, but it is sufficient to show the complications with which people will have to grapple before unambiguous measurements can be made. Briefly, Simpson's argument seems to have been based on the standard approximations in the calculation of the rate of $\beta$-decay which, among other things, suppose that the electron that escapes from the decaying nucleus has no effect on the electron of the tritium atoms (which is transformed by the decay into a singly ionized helium atom). But electrons are in- distinguishable on the scale of atoms, so may not the $\beta$-decay of tritium proceed by the capture of $\beta$-electron into a bound state and the transfer of the original bound electron into an essentially free state? Plainly the influence of these routes to $\beta$-decay will be greater when the electronenergy is small; Haxton argues that a first calculation shows them to be large enough to account for roughly one sixth of the departures from expectation reported by Simpson. Whatever the inevitable extrapolation of these arguments may bring, the result will be more accurate calculations of the detail of the decay of tritium than there have ever been before.

In the same spirit, whatever the immediate importance of the work of the Princeton group, the result will be a dramatic improvement in the accuracy of measurement of $\beta$-decay. Altzitzoglou et al. have designed what is in effect a dedicated equipment for the direct measurement of $\beta$-decay spectra. The trick is to build a cylindrical vacuum chamber with a pair of symmetrically-placed coils to provide an axial magnetic field, and to arrange for the accurate positioning of a radioactive source and an electrondetector at the opposite ends of the axis. The chamber is iron-free, decay electrons are prevented from making the straightline journey from source to detector by a lead obstacle in the centre, but can reach the detector if they happen to be orientated so as to intercept a circular annulus cut in an otherwise impenetrable disk, also concentrically mounted. The detector will then see only those electrons that leave the source with an energy or momentum exactly corresponding to the geome ${ }^{t} y$ of the equipment. The spectrum of a $\beta$-decay process is then determined by varying the current and recording the rate at which particles reach the detector.

The result is simply stated. For sulphur35 , the measured spectra fit better with the assumption that all neutrinos have zero-mass than with the particular result put forward by Simpson. On the face of things, neutrinos have no mass or, more precisely, such states of massive neutrinos as there may be play only a small part (or mix) in the states of the neutrinos emitted in $\beta$-decay. But this conclusion naturally applies only if neutrino masses are as large as suggested by Simpson; nothing so far attempted can exclude masses some orders of magnitude smaller than Simpson's $17.1 \mathrm{keV}$.
John Maddox 